\title{
First and Second Law of Thermodynamics at strong coupling
}

\author{
Udo Seifert \\ II. Institut für Theoretische Physik, Universität Stuttgart, 70550 Stuttgart, Germany
}

\begin{abstract}
For a small driven system coupled strongly to a heat bath, internal energy and exchanged heat are identified such that they obey the usual additive form of the first law. By identifying this exchanged heat with the entropy change of the bath, the total entropy production is shown to obey an integral fluctuation theorem on the trajectory level implying the second law in the form of a Clausius inequalilty on the ensemble level. In this Hamiltonian approach, the assumption of an initially uncorrelated state is not required. The conditions under which the proposed identification of heat is unique and experimentally accessible are clarified.
\end{abstract}

PACS numbers: 05.70.Ln

The thermodynamic analysis of a system coupled to a work source and a heat bath of temperature $T$ typically relies crucially on the assumption that the coupling to the bath is weak. If this condition is not met, partitioning the work $\mathcal{W}$ spent in a process into dissipated heat $\mathcal{Q}$ and an increase in internal energy of the system $\Delta \mathcal{E}$ in the form of a first law

$$
\mathcal{W}=\Delta \mathcal{E}+\mathcal{Q}
$$

leaves the question open whether at all, and, if so, in which form the interaction between system and bath is contained in the two terms on the right hand side. The same issue arises in the second law when it is written in the additive form as a Clausius inequality

$$
\Delta \mathcal{S}_{\text {tot }}=\Delta \mathcal{S}+\mathcal{Q} / T \geq 0
$$

splitting the total entropy change $\Delta \mathcal{S}_{\text {tot }}$ in one of the system $\Delta \mathcal{S}$ and one of the bath given by the heat divided by $T$.

Work is arguably the least problematic of the five quantities appearing above since it can easily be identified even in the presence of strong coupling. By treating system and bath including the interaction as one big closed system that evolves under a time-dependent Hamiltonian, the change of the latter from an initial state to a final one represents work. If the initial state is drawn from a canonical ensemble for the whole system, work is known to obey exact relations like the Jarzynski equality [1] and the Crooks relation [2] even in the presence of strong coupling [3] as, inter alia, many single molecule experiments over the last decade have demonstrated convincingly [4-9]. Since typical work values of even a few hundred $k_{B} T$ become tiny when divided by the number of molecules in the solution in contact with the biomolecule, the change in the interaction between bath and molecule is not necessarily negligible compared to those in internal energy of the molecule. The success of these experiments therefore rests partially on the fact that their interpretation does not require splitting the work into internal energy and heat for these strongly coupled system. On the other hand, for driven solid state devices, recent progess in ultra-sensitive calorimetry should soon make heat exchange directly accessible experimentally $[10,11]$.

Exploring the role of strong coupling for equilibrium thermodynamics has a long history going back, in the classical case, at least to Kirkwood's concept of a potential of mean force [12, 13], see, e.g., Ref. [14] for a recent analysis. For quantum systems, the role of strong coupling has been discussed in particular in the context of damped harmonic oscillators for quite some time $[15,16]$ with a recent emphasis on apparent anomalies like a negative specific heat [17]. How to formulate a consistent thermodynamics for a strongly coupled system under non-equilibrium conditions, like relaxation after an initial quench or genuine time-dependent driving, has found more attention lately for quantum systems than for classical ones. Various approaches and schemes are discussed [18-33] without arguably reaching a consensus yet on how to identify, beyond work, the terms in (1) and (2) uniquely.

Crucial aspects surface similarly in both frameworks, classical and quantum. One common subtle issue concerns entropy production since treating the full system as closed, which works so nicely for an identification of work, implies on the other hand that the total change of Gibbs, or Shannon, entropy (classically), or of the von Neumann entropy in the quantum case, remains strictly constant even under time-dependent driving. A positive entropy production results, however, if one ignores the correlations between system and bath, see, e.g., Ref. [22]. Even then, however, the identification of heat is not unique as, e.g., the comparison of two schemes for a simple relaxation for quantum Brownian motion has shown [27]. Moreover, in these approaches, one often assumes that initially system and bath are individually equilibrated as if there was no interaction. For most bio-molecular systems in aqueous solution, however, such an assumption is certainly rather unrealistic.

In this paper, we present an approach that allows to identify the terms appearing in the additive forms of the first and the second law consistently for driven classical systems strongly coupled to a heat bath without requiring an initially uncorrelated state. In the limit of 
weak coupling, these quantities will become the established ones. A particular virtue of this approach is that the terms appearing in (1) and (2) can be inferred from measurements involving only observables of the system.

As reference for the driven case, and to establish notation, we first recall the equilibrium situation, see, e.g., $[14,25]$. For a system coupled to a heat bath, the total Hamiltonian reads

$$
H_{\text {tot }}(\xi, \lambda)=H_{\mathrm{s}}\left(\xi_{\mathrm{s}}, \lambda\right)+H_{\mathrm{b}}\left(\xi_{\mathrm{b}}\right)+H_{\mathrm{i}}(\xi),
$$

comprising, in this order, system, bath, and interaction Hamiltonian. A micro state in the full phase space is written as $\xi \equiv\left(\xi_{\mathrm{s}}, \xi_{\mathrm{b}}\right)$ where $\xi_{\mathrm{s}}$ and $\xi_{\mathrm{b}}$ denote micro states in the phase space of system and bath, respectively. The control parameter $\lambda$, which will later be used to drive the system, does neither affect the bath nor the interaction part of the Hamiltonian. While in a Hamiltonian approach it may look more natural to consider a microcanonical equilibrium for the full system, for technical reasons that will become clear later we rather choose a canonical equilibrium for the total system at inverse temperature $\beta$. Then the probability to find the system part in a state $\xi_{\mathrm{s}}$ is given by

$$
p^{\mathrm{eq}}\left(\xi_{\mathrm{s}} \mid \lambda\right)=\exp \left[-\beta\left(\mathcal{H}\left(\xi_{\mathrm{s}}, \lambda\right)-\mathcal{F}(\lambda)\right)\right]
$$

Here,

$$
\mathcal{H}\left(\xi_{\mathrm{s}}, \lambda\right) \equiv H_{\mathrm{s}}\left(\xi_{\mathrm{s}}, \lambda\right)-\beta^{-1} \ln \left\langle\exp \left[-\beta H_{\mathrm{i}}(\xi)\right]\right\rangle_{\mathrm{b}}
$$

is an effective Hamiltonian, or, in the jargon of physical chemistry, a potential of mean force. It involves a canonical average over the pure bath (at fixed $\xi_{\mathrm{s}}$ ) denoted in the following by

$$
\langle\ldots\rangle_{\mathrm{b}} \equiv \int d \xi_{\mathrm{b}} \ldots \exp \left[-\beta\left(H_{\mathrm{b}}\left(\xi_{\mathrm{b}}\right)-\mathcal{F}_{\mathrm{b}}\right)\right]
$$

where $\mathcal{F}_{\mathrm{b}}$ is the free energy of the pure bath. The $\lambda$ dependent free energy of the system is defined through

$$
\exp [-\beta \mathcal{F}(\lambda)] \equiv \int d \xi_{\mathrm{s}} \exp \left[-\beta \mathcal{H}\left(\xi_{\mathrm{s}}, \lambda\right)\right]
$$

Still in equilibrium, this free energy implies through the standard relation $\mathcal{S}=\beta^{2} \partial_{\beta} \mathcal{F}$ for the entropy of the system

$$
\mathcal{S}(\lambda)=\int d \xi_{\mathrm{s}} p^{\mathrm{eq}}\left(\xi_{\mathrm{s}}\right)\left[-\ln p^{\mathrm{eq}}\left(\xi_{\mathrm{s}}\right)+\beta^{2} \partial_{\beta} \mathcal{H}\left(\xi_{\mathrm{s}}, \lambda\right)\right]
$$

setting Boltzmann's constant to 1 throughout. Likewise, the internal energy $\mathcal{E}=\mathcal{F}+\mathcal{S} / \beta$ becomes

$$
\mathcal{E}(\lambda)=\int d \xi_{\mathrm{s}} p^{\mathrm{eq}}\left(\xi_{\mathrm{s}}\right) E\left(\xi_{\mathrm{s}}, \lambda\right)
$$

with

$$
E\left(\xi_{\mathrm{s}}, \lambda\right) \equiv \mathcal{H}\left(\xi_{\mathrm{s}}, \lambda\right)+\beta \partial_{\beta} \mathcal{H}\left(\xi_{\mathrm{s}}, \lambda\right)
$$

In the weak coupling limit, the three energy functions $H_{s}, \mathcal{H}$, and $E$ converge.

The additional contribution $\sim \partial_{\beta} \mathcal{H}\left(\xi_{\mathrm{s}}, \lambda\right)$ beyond what one might have expected naively for entropy and internal energy takes into account that due to the finite interaction the bath is correlated with the microstate $\xi_{\mathrm{s}}$ of the system. In fact, with the standard canonical equilibrium for the total system obeying in obvious notation the relation $\mathcal{F}_{\text {tot }}=\mathcal{E}_{\text {tot }}-\mathcal{S}_{\text {tot }} / \beta$ and that for the pure bath with $\mathcal{F}_{\mathrm{b}}=\mathcal{E}_{\mathrm{b}}-\mathcal{S}_{\mathrm{b}} / \beta$ the above identified thermodynamic quantities of the system fulfill

$$
\mathcal{X}=\mathcal{X}_{\text {tot }}-\mathcal{X}_{\mathrm{b}}
$$

for $\mathcal{X}=\mathcal{F}, \mathcal{E}, \mathcal{S}$. This additive relation indicates that in this approach the interaction is fully accounted for through modification of the quantities refering to the system.

We now drive the system for a time $t$ through a timedependent control parameter $\lambda^{\tau}$, with $0 \leq \tau \leq t$. The total system comprising the system proper, the heat bath and the interaction is assumed to be closed. An initial phase point $\xi^{0}$ then evolves in time deterministically into $\xi^{t}$. The corresponding mapping $\xi^{t}=\xi^{t}\left(\xi^{0}\right)$ has Jacobian 1 due to Liouville's theorem. We first keep a trajectorybased approach $[34,35]$ in which all quantities become a function of the initial phase point $\xi^{0}$.

The work spent in the driving is the total energy difference

$$
w\left(\xi^{0}\right) \equiv \Delta H_{\text {tot }}(\xi, \lambda) \equiv H_{\text {tot }}\left(\xi^{t}, \lambda^{t}\right)-H_{\text {tot }}\left(\xi^{0}, \lambda^{0}\right) .
$$

Here, and in the following, $\Delta$ operating on a quantity implies the difference of this quantity between final and initial value. Hamiltonian dynamics implies that this work can also be written as

$$
w\left(\xi^{0}\right)=\int_{0}^{t} d \tau \partial_{\lambda} H_{\mathrm{s}}\left(\xi_{\mathrm{s}}^{\tau}, \lambda^{\tau}\right) \partial_{\tau} \lambda
$$

which is the form used in stochastic energetics [34, 35]. In fact, one could replace here $\partial_{\lambda} H_{\mathrm{s}}$ by either $\partial_{\lambda} \mathcal{H}$ or $\partial_{\lambda} E$ without changing the subsequent results since all three derivatives are the same.

As a key step in the present approach, motivated by (9), internal energy of the system along a driven trajectory $\xi^{\tau}$ is identified as $E\left(\xi_{\mathrm{s}}^{\tau}, \lambda\right)$, independent of the specific (and in any case unknown) value of the instantaneous bath coordinates $\xi_{\mathrm{b}}^{\tau}$. As we will show below, thus a consistent thermodynamic scheme arises. This assignment of internal energy implies the identification of dissipated heat as

$$
q\left(\xi^{0}\right)=w\left(\xi^{0}\right)-\Delta E\left(\xi_{\mathrm{s}}, \lambda\right)=\Delta\left[H_{\mathrm{tot}}(\xi, \lambda)-E\left(\xi_{\mathrm{s}}, \lambda\right)\right]
$$

It is instructive to show more explicitly how the interaction modifies the standard forms of the terms in the first law. Writing

$$
H_{\mathrm{i}}(\xi)=\left\langle H_{\mathrm{i}}(\xi)\right\rangle_{\mathrm{b}}+\delta H_{\mathrm{i}}(\xi)
$$


we separate the mean interaction, at fixed system coordinate $\xi_{\mathrm{s}}$, from its fluctuations $\delta H_{\mathrm{i}}(\xi)$. Similarly, the energy of the bath is split according to

$$
H_{\mathrm{b}}\left(\xi_{\mathrm{b}}\right)=\left\langle H_{\mathrm{b}}\left(\xi_{\mathrm{b}}\right)\right\rangle_{\mathrm{b}}+\delta H_{\mathrm{b}}\left(\xi_{\mathrm{b}}\right) .
$$

With (5) and (10), the change in internal energy then becomes after little algebra

$$
\begin{aligned}
\Delta E\left(\xi_{\mathrm{s}}, \lambda\right)= & \Delta\left[H_{\mathrm{s}}\left(\xi_{\mathrm{s}}, \lambda\right)+\left\langle H_{\mathrm{i}}(\xi)\right\rangle_{\mathrm{b}}+\right. \\
& \left.+\left\langle\delta H_{\mathrm{i}}(\xi) B_{\mathrm{i}}(\xi)\right\rangle_{\mathrm{b}}+\left\langle\delta H_{\mathrm{b}}\left(\xi_{\mathrm{b}}\right) B_{\mathrm{i}}(\xi)\right\rangle_{\mathrm{b}}\right]
\end{aligned}
$$

where

$$
B_{\mathrm{i}}(\xi) \equiv \exp \left[-\beta \delta H_{\mathrm{i}}(\xi)\right] /\left\langle\exp \left[-\beta \delta H_{\mathrm{i}}(\xi)\right]\right\rangle_{\mathrm{b}}
$$

Thus the average interaction is fully attributed to the internal energy, which, however, also picks up two more contributions from the fluctuations. Correspondingly, the heat (14) becomes

$$
\begin{aligned}
q\left(\xi^{0}\right)= & \Delta\left[H_{\mathrm{b}}\left(\xi_{\mathrm{b}}\right)+\delta H_{\mathrm{i}}(\xi)\right. \\
& \left.-\left\langle\delta H_{\mathrm{i}}(\xi) B_{\mathrm{i}}(\xi)\right\rangle_{\mathrm{b}}-\left\langle\delta H_{\mathrm{b}}\left(\xi_{\mathrm{b}}\right) B_{\mathrm{i}}(\xi)\right\rangle_{\mathrm{b}}\right] .
\end{aligned}
$$

Beyond the standard expression of dissipated heat, which is the change in energy of the bath $\Delta H_{\mathrm{b}}\left(\xi_{\mathrm{b}}\right)$, the first two further contributions depend on how much the interaction fluctuates for a fixed system state $\xi_{\mathrm{s}}$. The last contribution depends on correlations of the interaction with fluctuations of the bath. In the weak coupling limit, these additional contributions vanish since the interaction becomes negligible.

The first law is thus obeyed on the trajectory level by construction. It will remain valid on the ensemble level after averaging with, in principle, any initial distribution $p^{0}(\xi)$. As physically sensible initial distributions we will choose from now on

$$
p^{0}(\xi)=p_{1}^{0}\left(\xi_{\mathrm{s}}\right) p_{2}^{\mathrm{eq}}\left(\xi_{\mathrm{b}} \mid \xi_{\mathrm{s}}, \lambda^{0}\right)
$$

where

$$
p_{2}^{\mathrm{eq}}\left(\xi_{\mathrm{b}} \mid \xi_{\mathrm{s}}, \lambda\right) \equiv \frac{\exp \left[-\beta\left(H_{\mathrm{i}}(\xi)+H_{\mathrm{b}}\left(\xi_{\mathrm{b}}\right)-\mathcal{F}_{\mathrm{b}}\right)\right]}{\left\langle\exp \left[-\beta H_{\mathrm{i}}(\xi)\right]\right\rangle_{\mathrm{b}}} .
$$

The initial distribution of the system $p_{1}^{0}\left(\xi_{\mathrm{s}}\right)$ is arbitrary. For technical reasons, we require that it does not vanish anywhere on the phase space of the system. The bath is assumed to be equilibrated initially for any system state $\xi_{\mathrm{s}}$. In the following, averages with this initial distribution will be denoted by $\langle\ldots\rangle$. Note that with the option of an initially non-equilibrated system part relaxation towards equilibrium at constant control parameter, e.g., after a quench of the system, is covered by this framework as well. If $p_{1}^{0}\left(\xi_{\mathrm{s}}\right)$ is the equilibrium distribution (4), then the initial distribution (20) corresponds to the canonical equilibrium in the full phase space.

We now turn to checking the consistency of the proposed identification of heat with the additive form of the second law. As a technical tool, we will use the trivial but powerful identity, or integral fluctuation theorem (IFT),

$$
1=\int d \xi^{t} \rho\left(\xi^{t}\right)=\left\langle\exp \left[\ln \left[\rho\left(\xi^{t}\left(\xi^{0}\right)\right) / p^{0}\left(\xi^{0}\right)\right]\right]\right\rangle .
$$

Liouville's theorem ensures that this IFT is valid for any normalized function $\rho(\xi)$ provided the initial distribution $p^{0}(\xi)$ vanishes nowhere on the full phase space. By choosing the legitimate factorized form

$$
\rho(\xi)=p_{1}^{t}\left(\xi_{\mathrm{s}}\right) p_{2}^{\mathrm{eq}}\left(\xi_{\mathrm{b}} \mid \xi_{\mathrm{s}}, \lambda^{t}\right)
$$

where $p_{1}^{\tau}\left(\xi_{\mathrm{s}}\right)$ is the true marginal distribution for $\xi_{\mathrm{s}}$ at time $\tau$, the IFT (22) becomes after trivial algebra

$$
1=\left\langle\exp \left[-\left(\Delta s\left(\xi^{0}\right)+\beta q\left(\xi^{0}\right)\right)\right]\right\rangle
$$

where the average is over the initial distribution (20). Here, the change in system entropy along the trajectory is

$$
\Delta s\left(\xi^{0}\right) \equiv-\ln p_{1}^{t}\left(\xi_{\mathrm{s}}^{t}\right)+\ln p_{1}^{0}\left(\xi_{\mathrm{s}}^{0}\right)+\Delta \beta^{2} \partial_{\beta} \mathcal{H}\left(\xi_{\mathrm{s}}, \lambda\right) .
$$

The first two terms amount to the change in stochastic entropy familiar from stochastic thermodynamics [36]. The third contribution, called intrinsic entropy in a related context [37], has the same physical origin as discussed above in equilibrium. If we now identify, as usual, the entropy change of the bath on the trajectory level with the exchanged heat (times $\beta$ ), the exponent in (24) becomes the total entropy production,

$$
\Delta s_{\text {tot }}\left(\xi^{0}\right) \equiv \Delta s\left(\xi^{0}\right)+\beta q\left(\xi^{0}\right),
$$

which thus obeys an IFT

$$
\left\langle\exp \left[-\Delta s_{\text {tot }}\left(\xi^{0}\right)\right]\right\rangle=1 .
$$

Even though this IFT looks like the one derived earlier using a stochastic dynamics [36], one should note that here it follows from a Hamiltonian dynamics for a strongly coupled driven system.

The second law (2) for the calligraphic capitalized quantities that denote the averages with respect to the initial distribution (20) follows trivially from Jensen's inequality applied to (27). On a mathematical level, we have thus shown that if internal energy, heat, and the two contributions to total entropy production are identified as suggested here, the additive form of the first and second law are valid in the presence of strong coupling.

Can heat and the other quantities be measured in an experiment where one has access to the trajectory of the degrees of freedom of the system $\xi_{\mathrm{s}}^{\tau}$ but, of course, not to the bath coordinates? Equilibration at fixed $\lambda$ yields $\mathcal{H}\left(\xi_{\mathrm{s}}, \lambda\right)$ from measuring the corresponding equilibrium distribution (4). Repeating these measurements at a slightly different temperature will lead to $\partial_{\beta} \mathcal{H}\left(\xi_{\mathrm{s}}, \lambda\right)$ and thus to the internal energy $E\left(\xi_{\mathrm{s}}, \lambda\right)$ through (10). For 
the driven system, the work is accessible from observing the trajectory $\xi_{\mathrm{s}}^{\tau}$ using (13) since the $\lambda$-dependence of $H_{\mathrm{s}}\left(\xi_{\mathrm{s}}, \lambda\right)$ is controlled in an experiment. Hence, the heat can be inferred from evaluating (14). Finally, the change in system entropy follows from measuring the marginal distributions $p_{1}^{t}\left(\xi_{\mathrm{s}}\right)$ and $p_{1}^{0}\left(\xi_{\mathrm{s}}\right)$. Thus, all quantities are, at least in principle, measurable experimentally from trajectories $\xi_{\mathrm{s}}^{\tau}$ without ever having to measure a bath degree of freedom. The ensemble quantities appearing in (1) and (2) then follow from averaging the trajectoryresolved measurements.

A few further aspects, implications and perspectives are worth noting. First, is this assignment of heat, or, equivalently, internal energy unique? On a formal level, there seems to be freedom. Replacing internal energy, heat and change in system entropy on the trajectory level according to $E\left(\xi_{\mathrm{s}}, \lambda\right) \rightarrow E\left(\xi_{\mathrm{s}}, \lambda\right)+\chi\left(\xi_{\mathrm{s}}, \lambda\right), q \rightarrow$ $q-\Delta \chi\left(\xi_{\mathrm{s}}, \lambda\right)$, and $\Delta s \rightarrow \Delta s+\beta \Delta \chi\left(\xi_{\mathrm{s}}, \lambda\right)$, respectively, with an arbitrary system state function $\chi\left(\xi_{\mathrm{s}}, \lambda\right)$, which vanishes in the weak coupling limit, leaves the first law (14) and the IFT (27) invariant. In fact, the choice $\chi\left(\xi_{\mathrm{s}}, \lambda\right)=\mathcal{H}\left(\xi_{\mathrm{s}}, \lambda\right)-E\left(\xi_{\mathrm{s}}, \lambda\right)$ amounts to what has been discussed in Ref. [27] under the label "poised". The crucial point, however, is that any choice $\chi\left(\xi_{\mathrm{s}}, \lambda\right) \neq 0$ will spoil the thermodynamic relation $\mathcal{S}=\beta^{2} \partial_{\beta} \mathcal{F}$, or, equivalently, $d \mathcal{E}_{\mid \lambda}=T d \mathcal{S}_{\mid \lambda}$, when applied on the ensemble level to equilibrium. As long as one requires these latter relations for assigning the label "thermodynamically consistent" only the present scheme with $\chi\left(\xi_{\mathrm{s}}, \lambda\right) \equiv 0$ fulfills this criterion.

Second, we have assumed that the bath is in a systemstate dependent equilibrium initially. This choice is physically sensible if there is a separation of time-scales between system and bath. Even with such a separation, however, the Hamiltonian dynamics will not precisely lead to a distribution of the form (23) at time $t$. Using the latter in (22) should therefore be interpreted as a mathematical convenience for deriving the IFT (24) rather than as a statement about the true distribution. As an aside, note that substituting the canonical distribution of the full system at $\lambda^{t}$ for $\rho(\xi)$ into (22) yields the strongly coupled Jarzynski equality [3] for an initially equilibrated system in one line.

Third, equality in the second law usually requires a quasistatic process. In our approach, the second law (2) follows from the IFT (27). Any IFT requires for a saturation of the corresponding inequality that the underlying distribution for the exponent is delta-like. Thus equality in (2) holds if and only if $\Delta s_{\text {tot }}\left(\xi^{0}\right)$ vanishes identically for all initial micro states $\xi^{0}$. Ultimately, this requirement implies that the distribution for the full system starts and remains canonical throughout the process. In this respect, the strong coupling case does not differ from weak coupling. In fact, from a more physical perspective, one would expect that a moderate or strong coupling should facilitate equilibration and hence the realization of quasistatic conditions even more than the common idealized weak coupling case does.

Fourth, so far, we have not split the total volume into one of the system and one of the bath which would give rise to a pressure term. It would be interesting to explore which modifications arise from such a perspective in the case of strong coupling [38].

Finally, since the main part of this paper dealt with classical systems, it is worth emphasizing that the present scheme suggests, by analogy with (10), as an internal energy operator for the system in the quantum case

$\hat{E} \equiv-\left(1+\beta \partial_{\beta}\right)\left[\beta^{-1} \ln \operatorname{Tr}_{\mathrm{b}} \exp \left[-\beta\left(\hat{H}_{\mathrm{s}}+\hat{H}_{\mathrm{i}}+\hat{H}_{\mathrm{b}}-F_{\mathrm{b}}\right)\right]\right]$,

where hats denote operators, the trace is over the bath degrees of freedom and $\exp \left(-\beta F_{\mathrm{b}}\right) \equiv \operatorname{Tr}_{\mathrm{b}} \exp \left(-\beta \hat{H}_{\mathrm{b}}\right)$. In general, this operator $\hat{E}$ will be a quite complicated function of temperature and the parameters of the total Hamiltonian. The change in internal energy then follows, in principle, from two point measurements of $\hat{E}$ at $\tau=0$ and $\tau=t$. Since, in general, $\left[\hat{E}, \hat{H}_{\text {tot }}\right] \neq 0$, work as given by the difference in total energy can not be measured simultaneously. Hence, heat as the difference of work and internal energy is not accessible through this route in the quantum case.

In conclusion, for a classical driven system strongly coupled to a heat bath not only work but also internal energy, dissipated heat and entropy production can be identified on the level of a trajectory of the system. Total entropy production obeys an integral fluctuation theorem implying, on the ensemble level, a consistent interpretation of the second law as a Clausius inequality. For an experimental realization, the heat accompanying conformational changes of mechanically manipulated bio-molecules should be accessible experimentally through measurements at two different temperatures as suggested here. While the theory is not confined to this particular class, with such experiments these molecules could turn out to become one paradigm for studying heat exchange in small driven strongly coupled systems.

Acknowledgments: I thank S. Goldt and P. Pietzonka for a critical reading of this manuscript.

[1] C. Jarzynski, Phys. Rev. Lett. 78, 2690 (1997).

[2] G. E. Crooks, Phys. Rev. E 60, 2721 (1999).

[3] C. Jarzynski, J. Stat. Mech.: Theor. Exp. , P09005 (2004).

[4] J. Liphardt, S. Dumont, S. B. Smith, I. Tinoco Jr, and C. Bustamante, Science 296, 1832 (2002).

[5] D. Collin, F. Ritort, C. Jarzynski, S. Smith, I. Tinoco, and C. Bustamante, Nature 437, 231 (2005).

[6] N. C. Harris, Y. Song, and C. H. Kiang, Phys. Rev. Lett. 99, 068101 (2007).

[7] F. Ritort, Adv. Chem. Phys. 137, 31 (2008). 
[8] N. A. Gupta, V. Abhilash, K. Neupane, H. Yu, F. Wang, and M. T. Woodside, Nature Physics 7, 631 (2011).

[9] A. Alemany, A. Mossa, I. Junier, and F. Ritort, Nature Physics 8, 688-694 (2012).

[10] K. L. Viisanen, S. Suomela, S. Gasparinetti, O.-P. Saira, J. Ankerhold, and J. P. Pekola, New Journal of Physics 17, 055014 (2015).

[11] M. Campisi, J. Pekola, and R. Fazio, New Journal of Physics 17, 035012 (2015).

[12] J. G. Kirkwood, J. Chem. Phys. 3, 300 (1935).

[13] B. Roux and T. Simonson, Biophys. Chem. 78, 1 (1999).

[14] M. F. Gelin and M. Thoss, Phys. Rev. E 79, 051121 (2009).

[15] H. Grabert, U. Weiss, and P. Talkner, Z. Phys. B: Condens. Matter 55, 87 (1984).

[16] G. W. Ford, J. T. Lewis, and R. F. O'Connell, Phys. Rev. Lett. 55, 2273 (1985).

[17] G.-L. Ingold, P. Hänggi, and P. Talkner, Phys. Rev. E 79, 061105 (2009).

[18] T. M. Nieuwenhuizen and A. E. Allahverdyan, Phys. Rev. E 66, 036102 (2002).

[19] C. Hörhammer and H. Büttner, J. Stat. Phys. 133, 1161 (2008).

[20] M. Campisi, P. Talkner, and P. Hänggi, Phys. Rev. Lett. 102, 210401 (2009).

[21] I. Kim and G. Mahler, Phys. Rev. E 81, 011101 (2010).

[22] M. Esposito, K. Lindenberg, and C. V. den Broeck, New Journal of Physics 12, 013013 (2010).
[23] S. Deffner and E. Lutz, Phys. Rev. Lett. 107, 140404 (2011).

[24] S. Hilt, S. Shabbir, J. Anders, and E. Lutz, Phys. Rev. E 83, 030102 (2011)

[25] M. Campisi, P. Hänggi, and P. Talkner, Rev. Mod. Phys 83, 771 (2011).

[26] R. Kosloff, Entropy 15, 2100 (2013).

[27] L. Pucci, M. Esposito, and L. Peliti, Journal of Statistical Mechanics: Theory and Experiment 2013, P04005 (2013).

[28] R. Gallego, A. Riera, and J. Eisert, New Journal of Physics 16, 125009 (2014).

[29] J. Ankerhold and J. P. Pekola, Phys. Rev. B 90, 075421 (2014).

[30] M. Carrega, P. Solinas, A. Braggio, M. Sassetti, and U. Weiss, New Journal of Physics 17, 045030 (2015).

[31] G. D. Chiara, A. J. Roncaglia, and J. P. Paz, New Journal of Physics 17, 035004 (2015).

[32] P. Hänggi and P. Talkner, Nat. Phys. 11, 108 (2015).

[33] M. Esposito, M. A. Ochoa, and M. Galperin, Phys. Rev. Lett. 114, 080602 (2015).

[34] U. Seifert, Rep. Prog. Phys. 75, 126001 (2012).

[35] K. Sekimoto, Stochastic Energetics (Springer, Berlin, Heidelberg, 2010).

[36] U. Seifert, Phys. Rev. Lett. 95, 040602 (2005).

[37] U. Seifert, Eur. Phys. J. E 34, 26 (2011).

[38] C. Jarzynski, talk presented at the FQMT2015 in Prague. 